\title{
Sarcoidosis muscular en una paciente con hipercalcemia no mediada por PTH, reporte de un caso
}

\section{Fernando Rivera Toquica ${ }^{1}$, Henry Mauricio Arenas Quintero ${ }^{2}$} ${ }^{1}$ Médico, Residente de III año de Medicina Interna, Universidad Tecnológica
de Pereira
${ }^{2}$ Médico Endocrinólogo, Docente de cátedra posgrado Medicina Interna,
Universidad Tecnológica de Pereira, miembro de número Asociación
Colombiana de Endocrinología, Metabolismo y Diabetes, Director Clínica
de Tiroides clínica Comfamiliar Pereira, Risaralda.
Correspondencia: Dr. Henry Mauricio Arenas Quintero
E-mail: hmaq@utp.edu.co
Financiación: Para el desarrollo del manuscrito no se requirió nin-
guna fuente de financiación.
Conflicto de intereses: Ningún autor declara conflicto de intereses.

\section{Resumen}

$\mathrm{L}$ a sarcoidosis es una enfermedad granulomatosa sistémica de etiología desconocida, cuyos síntomas iniciales pueden ser inespecíficos y de intensidad variable, o incluso en muchos casos es asintomática. Cualquier órgano puede estar afectado, sin embargo, se observa con mayor frecuencia compromiso pulmonar y de ganglios linfáticos, seguido por la afectación a nivel oftálmico, cutáneo y hepático, el compromiso muscular aislado es poco frecuente. La sarcoidosis suele ser un diagnóstico de exclusión y en ocasiones puede imitar lesiones malignas, por lo que se debe considerar como un diagnóstico diferencial en muchas patologías, dada la gran variedad de manifestaciones. Presentamos el caso de una mujer de 34 años quien consultó por oligomenorrea, documentándose de manera incidental hipercalcemia no mediada por PTH, finalmente llegando al diagnóstico de una sarcoidosis muscular por medio de los hallazgos en el PET con 18-FDG, lo que permitió la realización de biopsia de músculo glúteo mayor izquierdo para confirmación histopatológica del diagnóstico.

Palabras clave: Sarcoidosis muscular, hipercalcemia, tomografía por emisión de positrones, reporte de caso.

\footnotetext{
Abstract

Sarcoidosis is a systemic granulomatous disease of unknown etiology, whose initial symptoms may be non-specific and variable in severity, or even asymptomatic in many cases. Any organ can be affected, but the lung and the lymphatic system are mostly involved, followed by the eyes, skin and liver, with isolated muscular involvement being rare. Sarcoidosis is an exclusion diagnosis and, in some cases, it could mimic malignancies, so it
}

should be considered in the differential diagnosis of many conditions, given its diverse manifestations. We present the case of a 34 years old female who complained of oligomenorrhea, with the incidental discovery of a non-PTH-mediated hypercalcemia. Finally, the diagnosis of muscular sarcoidosis was made using 18 FDG-PET followed by histopathology confirmation.

Keywords: Muscular sarcoidosis, hypercalcemia, positron emission tomography, case report.

\section{Introducción}

La sarcoidosis es una enfermedad sistémica de causa desconocida, en la cual un sujeto con predisposición genética es expuesto a un factor ambiental desencadenando una respuesta inmunitaria anormal. Su presentación es más común en mujeres y en ciertos grupos raciales, como el afroamericano y escandinavo. Puede ocurrir a cualquier edad, observándose generalmente en adultos menores de 50 años, con un pico de incidencia entre los 20 y 40 años ${ }^{(8)}$, tiene una prevalencia alrededor de 4,7 a 64 por 100.000 habitantes y una incidencia de 1,0 a 35 por 100.000 habitantes por año. Se caracteriza por la formación de granulomas inmunes no caseificantes en diferentes órganos, lo que le confiere un grado especial de dificultad al momento del diagnóstico dada la gran variedad de manifestaciones derivadas del compromiso multisistémico.

La sarcoidosis puede comprometer articulaciones, músculos y huesos, ocasionando una variedad de manifestaciones músculo-esqueléticas a través de diferentes mecanismos. Se estima que el músculo esquelético en la sarcoidosis está involucrado en alrededor del 50 al 80\% de los pacientes, pero menos del 5\% de los individuos presentan síntomas, por lo que en muchos de los casos puede no ser diagnosticada, el síntoma más común es la fatiga y la debilidad muscular proximal simétrica.

La hipercalcemia en la sarcoidosis se explica por la sobrexpresión de la enzima 1-alfa hidroxilasa por parte de los macrófagos presentes en los granulomas, su incidencia varía debido a diferentes factores tanto inherentes al huésped como

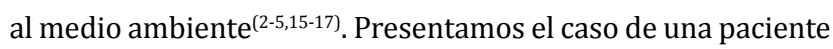
con sarcoidosis muscular generalizada asintomática detectada por tomografía por emisión de positrones (PET) con 18-fluorodeoxiglucosa (18-FDG) realizada durante el estudio de hipercalcemia no mediada por hormona paratiroidea (PTH). 


\section{Descripción del caso}

En junio de 2012, una mujer de 32 años procedente de la ciudad de Pereira, Risaralda, consulta a medicina general por un cuadro clínico de tres meses de evolución de pérdida involuntaria de 7 kilogramos de peso sin pérdida del apetito, además de oligomenorrea asociada a galactorrea, astenia y adinamia de dos meses de evolución. Dentro de sus antecedentes de importancia, había sido hospitalizada en octubre de 2008 por un cólico renal agudo, en esa oportunidad se documentó una ureterolitiasis distal izquierda por un cálculo de $2 \mathrm{~mm}$ el cual fue manejado de manera conservadora por parte de urología, dando la posibilidad de expulsión.

$\mathrm{Al}$ examen físico se encontraba una paciente alerta, consciente, sin signos de dificultad respiratoria en reposo, con una tensión arterial de 140/90 $\mathrm{mmHg}$, frecuencia cardiaca $85 \mathrm{lpm}$, frecuencia respiratoria $19 \mathrm{rpm}$, temperatura $36,4^{\circ} \mathrm{C}$, saturación de oxígeno $97 \%$, peso $59 \mathrm{~kg}$, talla 1,66 metros, índice de masa corporal $21,4 \mathrm{~kg} / \mathrm{m}^{2}$, mucosa oral húmeda, no soplos carotídeos, no masas, ni adenopatías, tiroides grado IA, móvil, blanda, no dolorosa a la palpación, mamas con galactorrea escasa a la expresión, ruidos respiratorios sin agregados, ambos campos pulmonares bien ventilados, extremidades sin edemas, fuerza $5 / 5$ y reflejos osteotendinosos $2 / 4$ en las 4 extremidades, no se evidencia déficit sensitivo; el resto del examen físico sin otros hallazgos de importancia.

Dentro de los paraclínicos iniciales del 21 de junio de 2012 se documentó creatinina: 1,60 mg/dL, prolactina: $123,9 \mathrm{ng} / \mathrm{mL}$ (valor de referencia 2 - $29 \mathrm{ng} / \mathrm{mL}$ ), TSH: 3,48 mUI/mL, 2 pruebas de embarazo negativas, uroanálisis con hematuria, por lo que se solicitó una ecografía renal y de vías urinarias que documentó pequeño cálculo de 3,3 mm en grupo calicial medio derecho sin evidencia de signos obstructivos. Un mes después fue valorada por ginecología donde se consideró dados los síntomas de la paciente: galactorrea sin estímulo y oligomenorrea con niveles elevados de prolactina, solicitar resonancia magnética de silla turca con gadolinio con el fin de descartar prolactinoma, la cual fue reportada dentro de límites normales con una hipófisis de 6,1 mm de diámetro, por lo que se descartó inicialmente lesión a ese nivel como causante del cuadro clínico actual. Concomitantemente fue valorada por nefrología por la presencia de hipertensión arterial (HTA) e insuficiencia renal (IR) de tiempo y evolución desconocida, solicitan estudios adicionales, reportando volumen de orina 2,1 litros, proteínas en orina de 24 horas: 163,8 mg/24h, proteínas totales: 7,9 g/dL, albúmina: 4,1 g/dL, globulinas: 3,32 g/dL, creatinina: 1,79 mg/dL, fósforo: $2,8 \mathrm{mg} /$ $\mathrm{dL}$ (VR 2,7-4,5 mg/dL), sodio $142 \mathrm{mEq} / \mathrm{L}$, potasio 4,3 mEq/L y calcio sérico: $15 \mathrm{mg} / \mathrm{dL}$ (VR 8,5-10,2 mg/dL). Ante la presencia de enfermedad renal crónica (ERC) e hipercalcemia verdadera (calcio sérico corregido por la albúmina 14,9 mg/dL y calcio iónico 1,51 mmol/l), es reevaluada por nefrología, considerando que la elevación leve de la prolactina posiblemente sea secun- daria a la ERC e inicia estudios para hipercalcemia. En agosto de 2012 la paciente asiste a control con reporte de PTH: 9,3 pg/ mL (VR: 15,0 - 65,0 pg/mL), calcio: 364,0 mg/24hrs (VR: 100,0 $321,0 \mathrm{mg} / 24 \mathrm{hrs}$ ), electroforesis de proteínas en suero sin picos monoclonales significativos excepto región gamma, concluyendo que la paciente cursaba con hipercalcemia no mediada por $\mathrm{PTH}$, por lo que nefrología consideró malignidad como primera opción. Se realiza endoscopia de vías digestivas altas, la cual fue negativa para malignidad y se decide remitir a endocrinología.

En septiembre de 2012 fue valorada por nuestro servicio de endocrinología donde consideramos que la paciente ameritaba ampliación del estudio hacia patología maligna vs granulomatosa dado que cursaba con hipercalcemia e hipercalciuria no mediadas por PTH, por lo que se solicitaron niveles de 25-hidroxivitamina D $\left(25[\mathrm{OH}] \mathrm{D}_{3}\right)$ y 1,25-dihidroxivitamina D $\left(1,25[\mathrm{OH}]_{2} \mathrm{D}_{3}\right)$, encontrando $25[\mathrm{OH}]_{3}: 38,8 \mathrm{ng} / \mathrm{mL}$ (normal) y $1,25[\mathrm{OH}]_{2} \mathrm{D}_{3}: 147 \mathrm{ng} / \mathrm{mL}$ (elevada) con un calcio sérico de 14,9 $\mathrm{mg} / \mathrm{dL}$, dados los hallazgos consideramos la posibilidad diagnóstica de linfoma o enfermedad granulomatosa como origen de la patología de la paciente, por lo que fue valorada de manera conjunta con hematología donde se consideró descartar gammapatía monoclonal ante el discreto aumento observado en la región gamma en la electroforesis de proteínas en suero (1,84 g/l, correspondiente al 23\%). Se le solicitaron niveles en suero de cadenas livianas kappa: 438,40 (VR: 138-375) y lambda: 280,10 (VR: 93-242), proteínas de Bence-Jones: negativas, inmunoglobulinas G, E, M: dentro de límites normales e inmunofijación de proteínas no detectables, por lo que se descartó posibilidad de mieloma múltiple, además biopsia y aspirado de médula ósea que no documentó infiltración de plasmocitos, médula normocelular, cariotipo 46XX, sin infiltración neoplásica. Ante la no evidencia del origen de la hipercalcemia, se decide hospitalizar para nefroprotección y realización de estudios tomográficos (Tomografía axial computarizada (TAC) de cuello, tórax, abdomen y pelvis), en los cuales no se evidenció compromiso pulmonar ni nodal relevante, así como tampoco se documentó hepatoesplenomegalia que orientara hacia un síndrome linfoproliferativo causante de la hipercalcemia, por lo que se descartó neoplasia hematolinfoide.

La paciente estuvo en observación durante 6 meses con calcio elevado, sin síntomas adicionales y sin pérdida de peso. Finalmente, ante la incertidumbre diagnóstica, manifestaciones de adinamia, astenia y cefalea global por parte de la paciente, función renal estable pero disminuida para la edad y calcio persistentemente elevado se solicitó un PET con 18-FDG, el cual reportó múltiples áreas hipermetabólicas que comprometían músculos en el esqueleto axial y apendicular sin alteraciones de la estructura anatómica, considerándose como posibilidad diagnóstica proceso granulomatoso o miopatía (figura 1), por lo que posteriormente se realizó biopsia de músculo glúteo mayor izquierdo que fue reportada como granuloma sarcoideo (figura 2). 
Figura 1. PET con 18-FDG (A) y (B). Muestra hipermetabolismo difuso en músculos del esqueleto axial y apendicular. (C) Sitio de mayor metabolismo a nivel de región glútea o músculo cuadriceps izquierdo (flecha blanca)

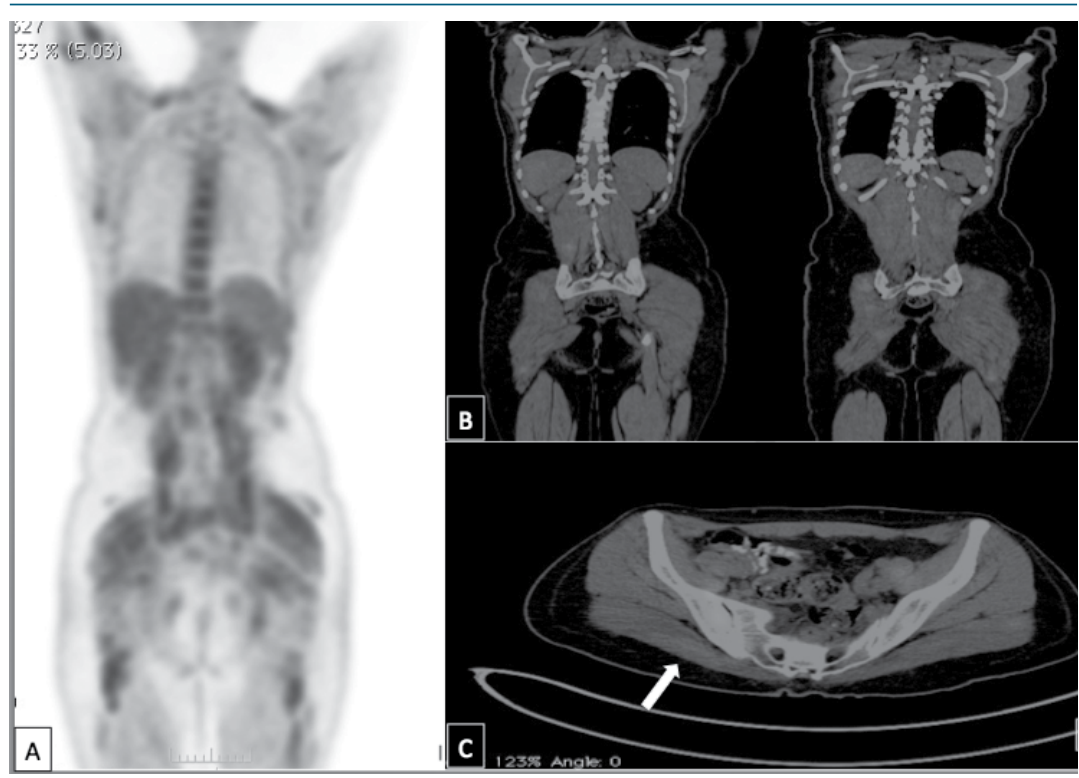

Figura 2. Biopsia de músculo glúteo mayor izquierdo. Fibras musculares (flechas negras gruesas). Hallazgos microscópicos demuestran inflamación granulomatosa con células gigantes multinucleadas (cabeza de flechas) la mayoría de ellas de cuerpo extraño con algunos cuerpos asteroides sin necrosis (flechas negras delgadas) y rodeadas por inflamación linfocítica con fibrosis alrededor que sugiere granuloma sarcoideo.

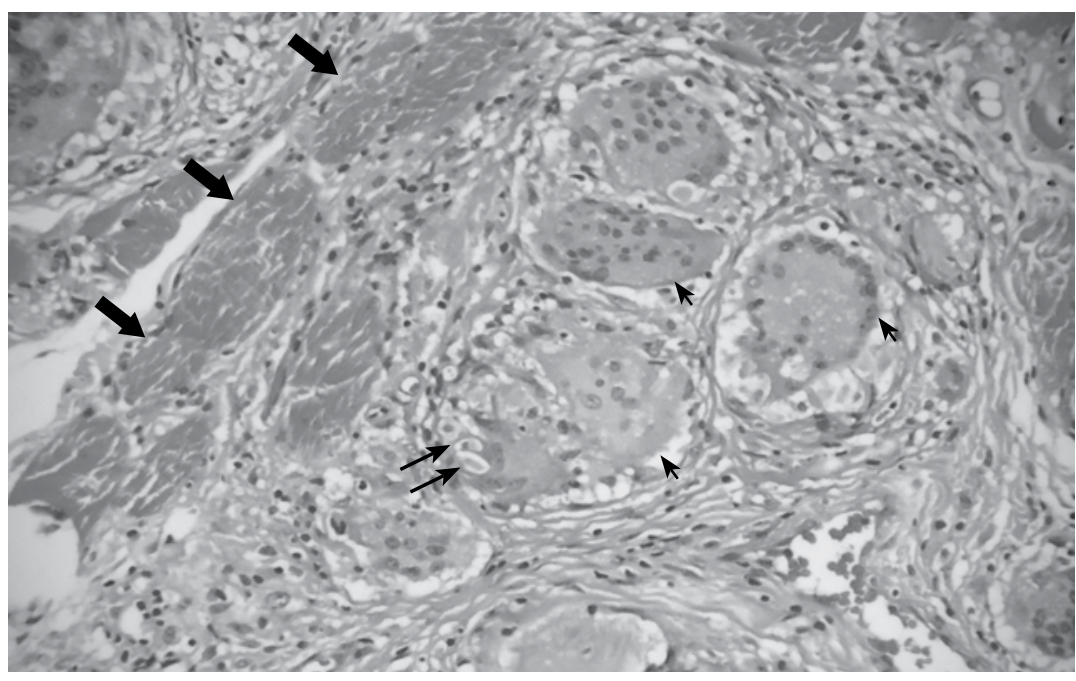

Se consideró que la hipercalcemia e hipercalciuria no mediada por PTH eran secundarias a una sarcoidosis muscular de tipo no específico, por lo que se inició manejo con prednisona 10 mg vía oral día, observando excelente respuesta con descenso progresivo de los niveles de calcio y creatinina (figura 3). Presentó como complicación de la inmunosupresión cuadro de reactivación de toxoplasmosis ocular tratada con clindamicina con adecuada respuesta.

\section{Discusión}

La prevalencia de la hipercalcemia depende de la población estudiada. La hipercalcemia persistente ha sido reportada hasta en $1 \%$ de individuos en la población general, y hasta en 3\% en pacientes hospitalizados. El hiperparatiroidismo primario es la causa más común de hipercalcemia en la población ambulatoria (54\%), de los cuales casi el $85 \%$ corresponden a un adenoma paratiroideo. En el caso de la población hospitalaria, la hipercalcemia secundaria a malignidad es la causa más común (50\%), el resto de causas son menos comunes (tabla 1) ${ }^{(10,20,21)}$. La hipercalcemia puede resultar debido a niveles inapropiadamente altos de PTH (hiperparatiroidismo primario), aumento de $1,25[\mathrm{OH}]_{2} \mathrm{D}_{3}$ (enfermedad granulomatosa y linfomas), péptido relacionado con la paratohormona (PTH-rp) (malignidad) u osteólisis local (malignidad).

Cada vez que evaluamos a un paciente con hipercalcemia, lo primero que debemos hacer es confirmar esta hipercalcemia con una segunda toma de calcio sérico y descartar la pseudohipercalcemia (por medio de la corrección del calcio sérico total con los niveles de albúmina). El objetivo es tratar de diferenciar la hipercalcemia mediada por la hormona paratiroidea de la hipercalcemia no mediada por ésta. Esto se hace mediante una evaluación clínica y paraclínica, en la cual se debe incluir: duración de la hipercalcemia, presencia o ausencia de síntomas, historia familiar, uso de medicamentos, y la medición de la PTH intacta, que es la prueba más importante en el diagnóstico diferencial de la hipercalcemia. Un valor elevado o normal/alto de PTH orienta hacia un hiperparatiroidismo primario, a diferencia de un valor bajo o normal/bajo que sugiere hipercalcemia no mediada por PTH, circunstancia en la que se indica la necesidad de evaluar otras causas menos comunes de hipercalcemia, incluyendo medición de PTH-rp y metabolitos de la vitamina $D$, etc. (figura 4).

La sarcoidosis fue descrita por primera vez en 1877 por el dermatólogo inglés J. Hutchinson ${ }^{(1)}$. Es una enfermedad multisistémica granulomatosa de causa desconocida, en la que se presume que, dada la frecuencia del compromiso de la piel, los ojos y los pulmones, la causa esté en el medio ambiente ${ }^{(9)}$. Se ha establecido un componente genético dentro de la fisiopa- 
Figura 3. Evolución paraclínica de la paciente. Inicio del tratamiento con esteroides (flecha), evidenciando posteriormente descenso del calcio iónico y de la creatinina.

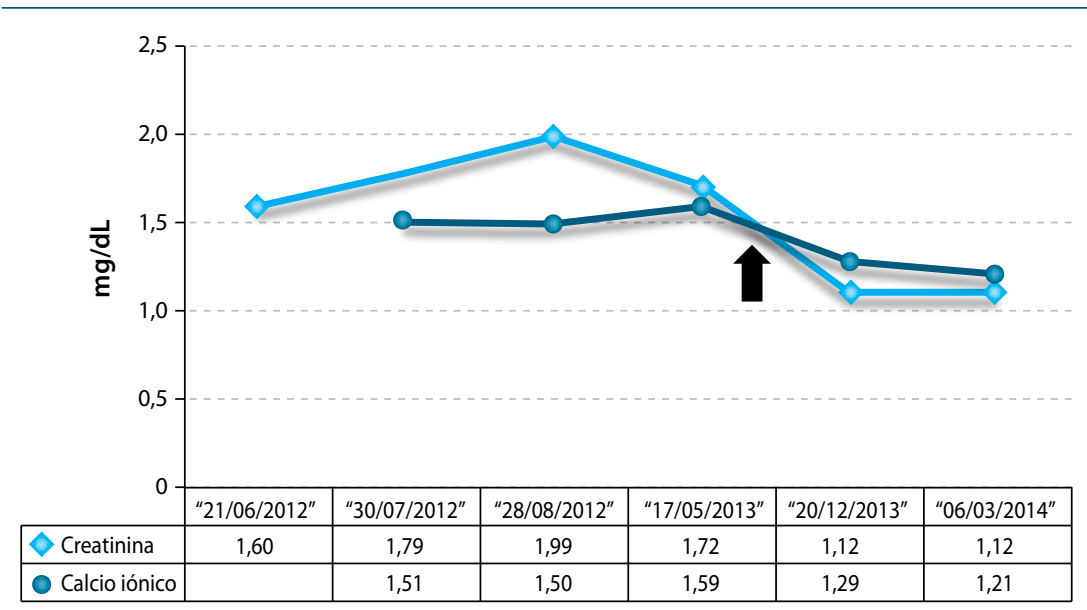

tología de la enfermedad, ya que ciertos alelos HLA parecen conferir susceptibilidad a la sarcoidosis, tales como HLA DR $11,12,14,15$ y 17 , mientras que otros como HLA DR1, DR4, y posiblemente HLA DQ*0202 confieren un efecto protector $^{(13)}$; sin embargo, la pesquisa de sarcoidosis entre los familiares no se recomienda, dado que menos del $1 \%$ desarrollan la enfermedad ${ }^{(11)}$. La sarcoidosis se caracteriza por la presencia de granulomas epitelioides no caseificantes, los cuales pueden aparecer en cualquier órgano del cuerpo. Los pulmones y los ganglios linfáticos intratorácicos son los órganos que con mayor frecuencia se ven afectados, casi en $90 \%$ de los pacientes, seguidos por compromiso a nivel of-
Tabla 1. Causas de hipercalcemia

Mediados por hormona paratiroidea (PTH)

- Hiperparatiroidismo primario (esporádico)

- Familiar

- Neoplasia endocrina múltiple (MEN) I o IIA

- Hipercalcemia hipocalciúrica familiar

- Hiperparatiroidismo terciario

- Malignidad coexistente e hiperparatiroidismo primario ectópico (Tumor productor de PTH).

\section{Cáncer}

- Hipercalcemia tumoral

- Mediada por péptido relacionado con la paratohormona (PTH-rp)

- Osteólisis local (metástasis óseas)

\section{Vitamina D}

- Enfermedad granulomatosa (producción extrarrenal de 1,25[OH] ${ }_{2} \mathrm{D}$ )

- Sarcoidosis, tuberculosis, beriliosis, coccidioidomicosis, histoplasmosis.

- Linfoma (producción extrarrenal de 1,25[OH $]_{2} \mathrm{D}$ )

- Intoxicación por vitamina D

- Suplementos de vitamina $D$, metabolitos o análogos de vitamina $D$ Falla renal

- Falla renal crónica con tratamiento con calcio y $1,25[\mathrm{OH}]_{2} \mathrm{D}_{3}$ o análogos de vitamina $D$

- Rabdomiólisis y falla renal aguda

- Trasplante renal (hiperparatiroidismo cuaternario)

Otros trastornos endocrinos

- Hipertiroidismo descompensado

- Insuficiencia adrenal

- Feocromocitoma

- Acromegalia

Medicamentos

- Diuréticos tipo tiazida

- Litio

- Teriparatide

- Intoxicación por vitamina A

- Otros fármacos: foscarnet, tamoxifeno, teofilina.

\section{Otros}

- Nutrición parenteral

- Síndrome de leche alcalina

- Inmovilización prolongada tálmico (11-83\%), hepático (75\% de las biopsias hepáticas en sarcoidosis), cutáneo (20-30\%), renal (7-22\%), cardiaco (317\%), esplénico (5-14\%), sistema nervioso central (5-10\%) y muscoloesquelético, el cual puede tener una afectación muy variable, dependiendo de si el compromiso es óseo, articular y/o muscular, oscilando entre 1 y $5 \%^{(3-7,15)}$.

Como se enunció previamente, la sarcoidosis es una enfermedad multisistémica inflamatoria que se inicia por la interacción entre un antígeno desconocido y una célula presentadora de antígenos que provoca una respuesta errónea de los linfocitos T CD4+. Los órganos afectados son infiltrados por linfocitos $\mathrm{T} \mathrm{CD} 4+$, posteriormente sobreviene una compleja cascada con otras células inflamatorias, especialmente macrófagos, que llevan a la formación de granulomas y distorsión del microparénquima normal. Los granulomas inmunes se caracterizan por tener un centro folicular compuesto por células epitelioides y células T CD4 de tipo 1 (Th1), rodeados por un anillo de fibroblastos, células B y linfocitos T CD8. Los macrófagos activados se asemejan a las células epiteliales (de ahí su nombre de granulomas epitelioides). Estas células son la fuente principal de producción de enzima convertidora de angiotensina (ECA); si bien, ésta puede estar elevada en $60 \%$ de las sarcoidosis agudas y en $10 \%$ de pacientes con enfermedad crónica, sus niveles aislados no son específicos ni sensibles para el diagnóstico de sarcoidosis, ni tampoco son útiles en el seguimiento. A su vez, estas células son las culpables de que entre 10 y $20 \%$ de los pacientes con sarcoidosis presenten hipercalcemia, gracias a que estos macrófagos sobrexpresan la enzima 1-alfa hidroxilasa de manera independiente al mecanismo de retroalimentación negativa, favoreciendo la conversión de 25-hidroxivitamina D (25[OH]D) a 1,25-dihidroxivitamina $\mathrm{D}\left(1,25[\mathrm{OH}]_{2} \mathrm{D}_{3}\right)$; esta actividad enzimática autónoma conlleva a una sobreproducción de $1,25[\mathrm{OH}]_{2} \mathrm{D}_{3}$ (calcitriol) lo que incrementa la absorción intestinal del calcio de la dieta y 
Figura 4. Diagnóstico diferencial de la hipercalcemia

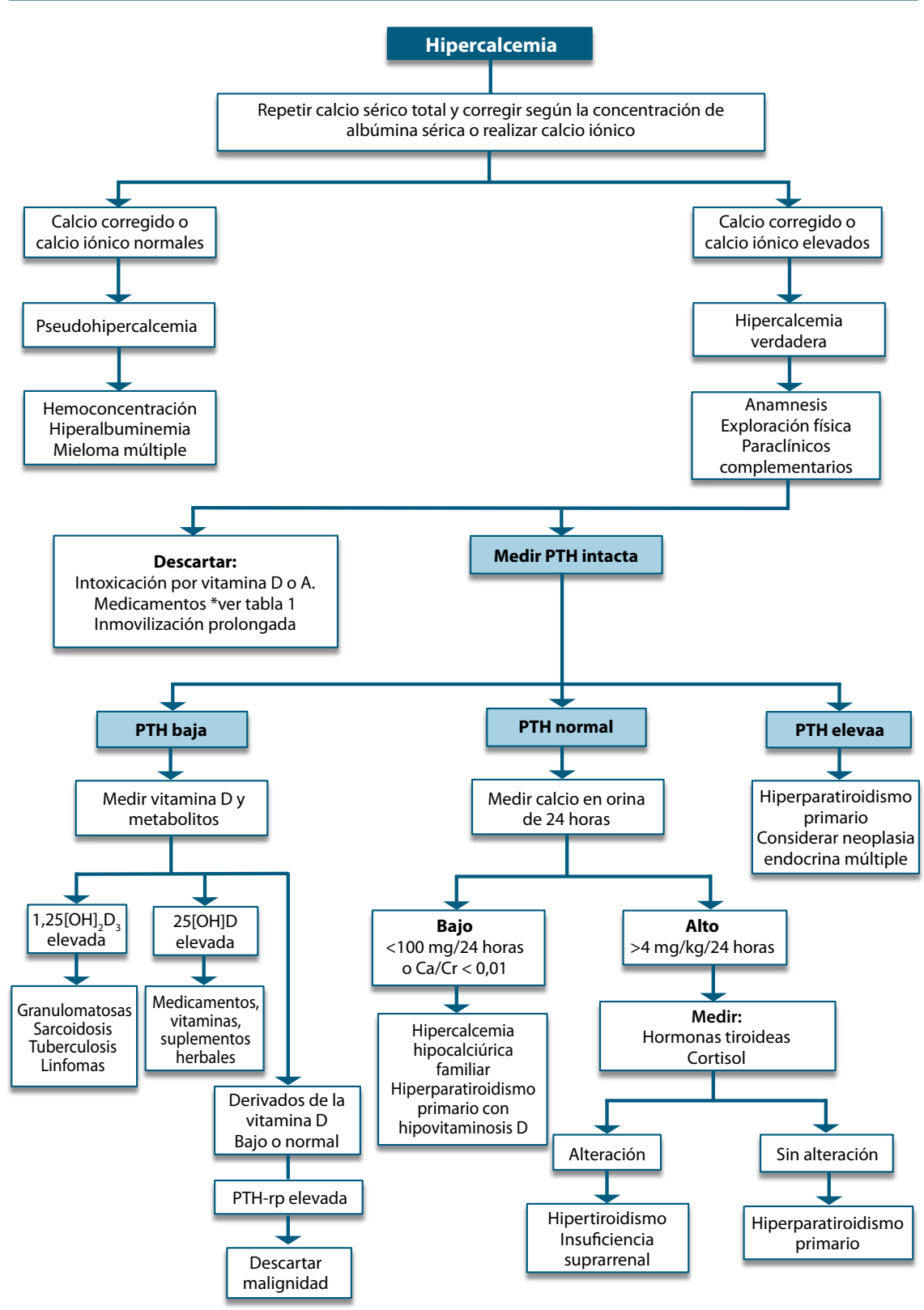

En la sarcoidosis un gran porcentaje de pacientes nunca experimentan manifestaciones, y en más de la mitad de los pacientes la remisión se produce dentro de los 3 primeros años, y sólo alrededor del 25\% desarrollan una enfermedad crónica $^{(8,9)}$. En el caso de la sarcoidosis muscular se estima que el músculo esquelético está involucrado en alrededor del $50 \%$ al $80 \%$ de los pacientes, pero menos del $5 \%$ de los individuos presentan síntomas, por lo que en muchos de los casos puede no ser diagnosticada. En la sarcoidosis muscular se pueden distinguir 3 subtipos: miopatía crónica, que es la forma más común de presentación de miopatía sarcoide, caracterizada por la presencia de mialgias, debilidad proximal simétrica y atrofia muscular, pudiendo encontrar a menudo los niveles de creatina cinasa (CK) normales, pero en muchos casos éstos pueden estar marcadamente elevados. Otra forma poco común de miopatía por sarcoidosis es la nodular palpable, caracterizada por acumulación de numerosos granulomas y tejido conectivo denso en los nódulos, clínicamente pueden ser muy dolorosas, pero raramente causan debilidad, usualmente son bilaterales y multifocales con cierta predilección por las uniones miotendinosas. Finalmente la miositis aguda es la forma menos común de las tres, se presenta con debilidad proximal de instauración rápida, mialgias y fiebre y con niveles de CK usualmente elevados ${ }^{(4,18)}$.

A pesar de una historia clínica y hallazgos físicos compatibles con sarcoidosis, en la mayoría de los casos es necesaria la confirmación histopatoló-

la actividad de los osteoclastos con un aumento en la resorción ósea y reabsorción de calcio a nivel renal consecuentemente, llevando a la generación de hipercalcemia.

La incidencia puede variar dependiendo de factores genéticos, exposición solar, el calcio de la dieta y el color de la piel, observándose mayor incidencia durante el verano y en hombres de raza blanca mayores de 40 años. La hipercalciuria es más común que la hipercalcemia, presentándose hasta en la mitad de los pacientes con sarcoidosis. Estas alteraciones metabólicas pueden llevar a nefrolitiasis en 10 a 15\% de los pacientes, nefrocalcinosis, depósitos intersticiales de calcio e insuficiencia renal como en el caso de nuestra paciente ${ }^{(4,5,9,12,14)}$. gica donde se evidencien los granulomas no caseificantes. Los sitios preferidos para biopsia de tejido son los periféricos como los ganglios linfáticos, la piel o la glándula parótida. El eritema nodoso a pesar de ser una lesión periférica, y frecuente en la sarcoidosis, que denota enfermedad aguda, no es una lesión recomendada para toma de biopsia como confirmación, ya que mostraría paniculitis y no granulomas.

Se consideran dos escenarios en los cuales ante la sospecha clínica se podría excluir la biopsia de tejido. La primera posibilidad es en el caso de que la sarcoidosis se presente como síndrome de Löfgren (fiebre, artralgias, adenopatías hiliares bilaterales, con o sin eritema nodoso), en el que se podría ex- 
cluir si los síntomas se resuelven de manera rápida y espontánea. En segundo lugar cuando el lavado broncoalveolar sea compatible con sarcoidosis (disminución en las células CD8+ e incremento en la relación CD4+/CD8+) ${ }^{(14)}$; en el resto de los casos como ya hemos referido siempre se debe llegar a la confirmación histopatológica por biopsia.

A pesar de que la paciente presentaba predictores de enfermedad crónica y de peor pronóstico como lo eran la hipercalcemia crónica y la miosarcoidosis ${ }^{(12,13)}$, presentó una buena respuesta a la terapia con corticoides orales, los cuales estaban indicados por la presencia de hipercalcemia, ya que consiguen disminuir los niveles de calcio sérico en pocos días, logrando el control de la producción de calcitriol a nivel del granuloma y mejorando así la tasa de filtración glomerular y el aclaramiento de creatinina. La dosis inicial de prednisona es de 20-40 mg/día y, según la evolución clínica, la dosis de mantenimiento oscila entre 5 y $10 \mathrm{mg} /$ día; la hidroxicloroquina también puede ser útil en el manejo de la hipercalcemia en dosis de $200-400 \mathrm{mg} /$ día, recordando que estos pacientes deben ser valorados cada 6 a 12 meses por oftalmología por el riesgo de retinopatía. En los casos de hipercalcemia persistente, el ketoconazol puede ser una opción terapéutica, ya que inhibe la 1-alfa hidroxilasa producida por los macrófagos en el granuloma sarcoideo y, por ende, inhibe la conversión de 25[OH]D a calcitriol ${ }^{(5,4,15,19)}$.

\section{Conclusiones}

Las manifestaciones extrapulmonares de la sarcoidosis constituyen en conjunto un sitio frecuente de compromiso. En el caso de nuestra paciente la cual consultó inicialmente por cuadro de oligomenorrea con galactorrea con prolactina elevada probablemente por compromiso hipofisiario previo por sarcoidosis?, o secundaria al compromiso renal, fue solo gracias al hallazgo incidental de hipercalcemia no mediada por PTH y los posteriores estudios que documentaron niveles elevados de $1,25[\mathrm{OH}]_{2} \mathrm{D}_{3}$ con lo que se pudo establecer una sospecha diagnóstica, logrando por medio del PET con 18-FDG determinar el compromiso muscular y posterior corroboración por medio de biopsia.

El compromiso renal por sarcoidosis que pudiera explicar la elevación de la prolactina también se encuentra en 10 a $20 \%$ de los pacientes en donde se puede evidenciar nefritis túbulointersticial, hipercalcemia, hipercalciuria, nefrocalcinosis y disminución de la tasa de filtrado glomerular, sin embargo en el caso de nuestra paciente la captación con 18 FDG en el PET no mostró alteración renal alguna, como se describe en reportes de sarcoidosis renal activa ${ }^{(22)}$. El PET con 18-FDG adquiere no sólo un valor diagnóstico inmenso en los casos de sarcoidosis de localización desconocida, sino que nos podría ayudar para monitorizar la actividad y la respuesta al tratamiento de la enfermedad ${ }^{(23)}$.
Toda hipercalcemia no mediada por PTH debe ser investigada aun con exámenes diagnósticos en ocasiones no específicos (p. ej. PET), pues como en este caso los síntomas y signos iniciales no enfocaban hacia esta patología y las causas pueden ser muy poco frecuentes como en la paciente presentada en este caso.

\section{Referencias}

1. Hutchinson J. Anomalous diseases of skin and fingers: case of livid 3 papillary psoriasis? In: Illustrations of clinical surgery. London: J and A Churchill, 1877:42-3.

2. Rybicki BA, Major M, Popovich J, Jr., Maliarik MJ, Iannuzzi MC. Racial differences in sarcoidosis incidence: a 5-year study in a health maintenance organization. American Journal of Epidemiology. 1997;145(3):234-41.

3. Baughman RP, Teirstein AS, Judson MA, Rossman MD, Yeager H, Jr., Bresnitz EA, et al. Clinical characteristics of patients in a case control study of sarcoidosis. American Journal of Respiratory and Critical Care Medicine. 2001;164(10 Pt 1):1885-9.

4. Rao DA, Dellaripa PF. Extrapulmonary manifestations of sarcoidosis. Rheumatic diseases clinics of North America. 2013;39(2):277-97.

5. Holmes J, Lazarus A. Sarcoidosis: extrathoracic manifestations. Disease-amonth : DM. 2009;55(11):675-92.

6. Haimovic A, Sanchez M, Judson MA, Prystowsky S. Sarcoidosis: a comprehensive review and update for the dermatologist: part I. Cutaneous disease. Journal of the American Academy of Dermatology. 2012;66(5):699.e1-18; quiz 717-8.

7. Siltzbach LE, James DG, Neville E, Turiaf J, Battesti JP, Sharma OP, et al. Course and prognosis of sarcoidosis around the world. The American Journal of Medicine. 1974;57(6):847-52.

8. O'Regan A, Berman JS. Sarcoidosis. Annals of Internal Medicine. 2012;156(9):ITC 5-1

9. Iannuzzi MC, Fontana JR. Sarcoidosis: clinical presentation, immunopathogenesis, and therapeutics. JAMA : the journal of the American Medical Association. 2011;305(4):391-9.

10. Endres DB. Investigation of hypercalcemia. Clinical biochemistry 2012;45(12):954-63.

11. Rybicki BA, Iannuzzi MC, Frederick MM, Thompson BW, Rossman MD, Bresn itz EA, et al. Familial aggregation of sarcoidosis. A case-control etiologic study of sarcoidosis (ACCESS). American Journal of Respiratory and Critical Care Medicine. 2001;164(11):2085-91.

12. Dempsey OJ, Paterson EW, Kerr KM, Denison AR. Sarcoidosis. BMJ (Clinica research ed). 2009;339:b3206.

13. Heinle R, Chang C. Diagnostic criteria for sarcoidosis. Autoimmunity Reviews. 2014;13(4-5):383-7.

14. Iannuzzi MC, Rybicki BA, Teirstein AS. Sarcoidosis. The New England Journal of Medicine. 2007;357(21):2153-65.

15. Valeyre D, Prasse A, Nunes H, Uzunhan Y, Brillet PY, Muller-Quernheim J. Sarcoidosis. Lancet. 2014;383(9923):1155-67.

16. Spagnolo P, Luppi F, Roversi P, Cerri S, Fabbri LM, Richeldi L. Sarcoidosis: challenging diagnostic aspects of an old disease. The American Journal of Medicine. 2012;125(2):118-25.

17. Silverstein A, Siltzbach LE. Muscle involvement in sarcoidosis. Asymptomatic, myositis, and myopathy. Archives of Neurology. 1969;21(3):235-41.

18. Vardhanabhuti V, Venkatanarasimha N, Bhatnagar G, Maviki M, Iyengar S, Adams WM, et al. Extra-pulmonary manifestations of sarcoidosis. Clinical Radiology. 2012;67(3):263-76.

19. Eklund A, du Bois RM. Approaches to the treatment of some of the troublesome manifestations of sarcoidosis. Journal of Internal Medicine. 2014;275(4):335-49.

20. Chang WT, Radin B, McCurdy MT. Calcium, magnesium, and phosphate abnormalities in the emergency department. Emergency Medicine Clinics of North America. 2014;32(2):349-66.

21. Civitelli R, Ziambaras K. Calcium and phosphate homeostasis: concerted interplay of new regulators. Journal of Endocrinological Investigation. 2011;34(7 Suppl):3-7.

22. Braun J,Kessler R, Constantinesco A, Imperiale A. 18F-FDG PET/CT in sarcoidosis management: review and report of 20 cases. Eur J Nucl Med Mol Imaging. 2008 Aug;35(8):1537-43.

23. Treglia G, Taralli S, Giordano A. Emerging role of whole-body 18F-fluorodeoxyglucose positron emission tomography as a marker of disease activity in patients with sarcoidosis: a systematic review. Sarcoidosis, vasculitis, and diffuse lung diseases : official journal of WASOG / World Association of Sarcoidosis and Other Granulomatous Disorders. 2011;28(2):87-94. 